\title{
Noncytotoxic orange and red/green derivatives of DsRed-Express2 for whole-cell labeling
} Rita L Strack ${ }^{1}$, Dibyendu Bhattacharyya ${ }^{2}$, Benjamin S Glick ${ }^{2}$ and Robert J Keenan*1

\author{
Address: ${ }^{1}$ Department of Biochemistry and Molecular Biology, The University of Chicago, Chicago, Illinois 60637, USA and ${ }^{2}$ Department of \\ Molecular Genetics and Cell Biology, and Institute for Biophysical Dynamics, The University of Chicago, Chicago, Illinois 60637, USA \\ Email: Rita L Strack - strackr@uchicago.edu; Dibyendu Bhattacharyya - dibyendu@uchicago.edu; Benjamin S Glick - bsglick@uchicago.edu; \\ Robert J Keenan* - bkeenan@uchicago.edu \\ * Corresponding author
}

This article is available from: http://www.biomedcentral.com/I472-6750/9/32

(C) 2009 Strack et al; licensee BioMed Central Ltd.

This is an Open Access article distributed under the terms of the Creative Commons Attribution License (http://creativecommons.org/licenses/by/2.0), which permits unrestricted use, distribution, and reproduction in any medium, provided the original work is properly cited.

\begin{abstract}
Background: Whole-cell labeling is a common application of fluorescent proteins (FPs), but many red and orange FPs exhibit cytotoxicity that limits their use as whole-cell labels. Recently, a tetrameric red FP called DsRed-Express2 was engineered for enhanced solubility and was shown to be noncytotoxic in bacterial and mammalian cells. Our goal was to create derivatives of this protein with different spectral properties.
\end{abstract}

Results: Building on previous studies of DsRed mutants, we created two DsRed-Express2 derivatives: E2-Orange, an orange FP, and E2-Red/Green, a dual-color FP with both red and green emission. We show that these new FPs retain the low cytotoxicity of DsRed-Express2. In addition, we show that these new FPs are useful as second or third colors for flow cytometry and fluorescence microscopy.

Conclusion: E2-Orange and E2-Red/Green will facilitate the production of healthy, stably fluorescent cell lines and transgenic organisms for multi-color labeling studies.

\section{Background}

Fluorescent proteins (FPs) are useful as whole-cell labels. For this purpose, FPs can be either monomeric or oligomeric. However, oligomeric FPs are often better for wholecell labeling because they tend to be brighter and more photostable than their monomeric counterparts [1].

Even if an FP has desirable fluorescence properties, it may have limited utility as a cellular label due to cytotoxicity at high expression levels [2-4]. Cytotoxicity has been observed with many red and orange FPs in both bacterial and mammalian cells [5]. Recently, we described a tetra- meric DsRed variant called DsRed-Express2 that is ideally suited to whole-cell labeling due to its minimal cytotoxicity, fast maturation, and high photostability [5]. To create DsRed-Express2, we mutated the surface of DsRed-Express (also known as DsRed.T1) [6] to decrease higher-order aggregation of the tetramers. These mutations allowed DsRed-Express2 to be well tolerated when expressed at high levels.

Here, we have modified the interior of DsRed-Express 2 to create two additional FPs that are useful for whole-cell labeling. The first new FP, E2-Orange, exhibits orange flu- 
orescence similar to that of previously described orange FPs [7-10]. E2-Orange matures quickly, and is substantially less cytotoxic and more photostable than other available orange FPs. The second new FP, E2-Red/Green, emits both red and green fluorescence, and can be distinguished from pure red or pure green FPs. E2-Orange and E2-Red/Green will be particularly useful for multi-color whole-cell labeling.

\section{Results and discussion An orange derivative of DsRed-Express2}

Orange FPs can be useful alone, in two-color studies with green FPs, or in three-color studies with green and far-red FPs. The previously available orange FPs include the oligomeric Kusabira-Orange (KO) [9], a monomeric derivative of $\mathrm{KO}$ called $\mathrm{mKO} 2$ [8], and a monomeric orange variant of DsRed called mOrange2 [10]. To engineer an orange derivative of DsRed-Express2, we mutated the first residue of the chromophore, glutamine- 66 , to threonine. In mOrange, threonine at position 66 drives formation of a third heterocycle (oxazole ring) in the chromophore, leading to blue-shifted spectra relative to DsRed $[7,11]$. Introduction of the same Q66T mutation into DsRed-Express2 resulted in blue-shifted excitation and emission maxima, indicating that the same chromophore cyclization chemistry can occur in the DsRed-Express2 interior.

DsRed-Express2 + Q66T was then subjected to random mutagenesis to identify brightening mutations. We identified two such mutations, V71A and S179T. Both mutations produced modest increases in extinction coefficient and quantum yield, and the S179T mutation also accelerated maturation. These mutations were combined to yield the final orange variant, E2-Orange [GenBank: FJ498891].

E2-Orange has excitation and emission maxima at 540 $\mathrm{nm}$ and $561 \mathrm{~nm}$, respectively (Figure 1A). As with DsRedExpress2, a substantial fraction of the fully mature E2Orange molecules contain a blue-absorbing and greenemitting chromophore (Figure 1B). However, excitation with blue light does not produce significant green fluorescence, presumably due to efficient intra-tetramer Förster resonance energy transfer (FRET). The presence of two chromophore species explains why E2-Orange has a lower extinction coefficient than other orange FPs when excited with yellow light (Table 1). When excited with blue light, E2-Orange is comparable in brightness to other orange FPs (data not shown).

E2-Orange matures quickly and is photostable (Table 1). Compared to previously available orange FPs, E2-Orange matures much faster than mOrange 2 or $\mathrm{KO}$ and about as fast as $\mathrm{mKO} 2$, with a half-time of $1.3 \mathrm{~h}$ at $37^{\circ} \mathrm{C}$ (Figure 1C). We measured photostability with a simple assay involving a fixed illumination intensity [5], and found that E2-Orange is more photostable than any of the other
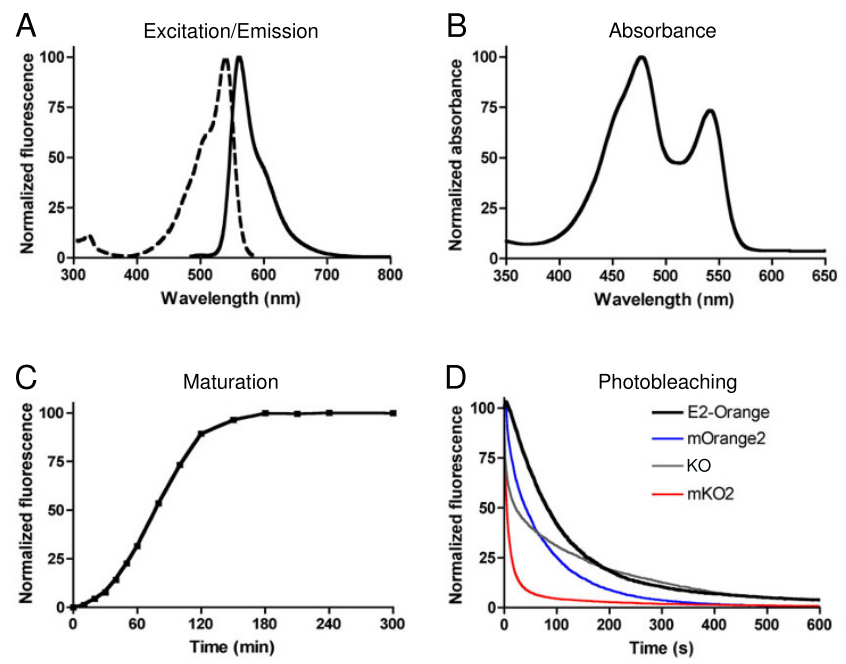

Figure I

Fluorescence properties of E2-Orange. Shown are (A) excitation and emission and (B) absorbance spectra of E2Orange. (C) Maturation kinetics of E2-Orange fluorescence. For these measurements the FPs were excited at $520 \pm 10$ $\mathrm{nm}$ excitation and emission was recorded at $560 \pm 10 \mathrm{~nm}$. (D) Photobleaching kinetics of E2-Orange (black line), mOrange2 (blue line), KO (gray line), and mKO2 (red line) during widefield fluorescence microscopy. Data were collected using a Texas Red filter set. For (C) and (D), data represent the mean of three independent measurements.

orange FPs tested (Table 1, Figure 1D). E2-Orange has a pKa of 4.5, making it the least acid-sensitive of the orange FPs tested (Table 1). Thus, the fluorescence properties of E2-Orange are favorable for whole-cell labeling.

To demonstrate the usefulness of E2-Orange in two-color labeling studies, the budding yeast Saccharomyces cerevisiae was transformed with vectors for high-level expression of either enhanced GFP (EGFP) or E2-Orange. By flow cytometry, these two populations of cells could be readily distinguished from each other and from cells not expressing an FP (Figure 2A).

To demonstrate the usefulness of E2-Orange as a third color in fluorescence microscopy, a S. cerevisiae strain was engineered to express cytosolic E2-Orange, plus Sec7EGFP to mark the late Golgi with green fluorescence [12], plus pCoxIV-mCherry to mark mitochondria with moderately far-red fluorescence $[6,7]$. The EGFP and E2-Orange signals were easily resolvable, and the E2-Orange and mCherry signals could be separated using linear unmixing, yielding clear three-color images (Figure 2B). The need for linear unmixing indicates that FPs further redshifted than mCherry (emission maximum $=610 \mathrm{~nm}$ ) will be most useful for three-color imaging with E2Orange and GFP. 
Table I: Properties of FPs.

\begin{tabular}{|c|c|c|c|c|c|c|c|}
\hline Fluorescent Protein & $\begin{array}{l}\text { Excitation/Emission } \\
\text { maxima }(\mathrm{nm})\end{array}$ & $\begin{array}{l}\text { Extinction coefficient } \\
\left(\mathrm{M}^{-1} \mathrm{~cm}^{-1}\right)\end{array}$ & Quantum yield & $\begin{array}{c}\text { Relative } \\
\text { brightness }\end{array}$ & $\begin{array}{l}\text { Maturation half- } \\
\text { time }(\mathrm{h})\end{array}$ & $\begin{array}{l}\text { Photobleaching half- } \\
\text { time }(s)^{b}\end{array}$ & $\mathrm{pKa}$ \\
\hline DsRed-Express2c & $554 / 591$ & 35,600 & 0.42 & 0.41 & 0.7 & $64 \pm 4$ & 4.5 \\
\hline $\begin{array}{l}\text { E2-Red/Green } \\
\text { (green) }\end{array}$ & $484 / 498$ & 100,200 & 0.06 & 0.17 & 0.4 & $236 \pm 8^{d}$ & 4.0 \\
\hline $\begin{array}{c}\text { E2-Red/Green } \\
\text { (red) }\end{array}$ & $560 / 585$ & 53,800 & 0.67 & 0.98 & 1.2 & $93 \pm 3$ & 4.5 \\
\hline E2-Orange & $540 / 561$ & 36,500 & 0.54 & 0.54 & 1.3 & $81 \pm 3$ & 4.5 \\
\hline mOrange2 & $549 / 563$ & 56,300 & 0.49 & 0.75 & 4.5 & $40 \pm 3$ & 7.5 \\
\hline KO & $548 / 560$ & 72,800 & 0.55 & I.I & 3.8 & $21 \pm 2$ & 5.0 \\
\hline $\mathrm{mKO}^{\mathrm{c}}$ & $549 / 563$ & 54,300 & 0.41 & 0.54 & 1.2 & $9 \pm 1$ & 5.0 \\
\hline
\end{tabular}

Unless otherwise indicated, all measurements were carried out during the present study using standardized procedures.

a Brightness was calculated as the product of extinction coefficient and quantum yield, and was normalized to a value of I for wild-type DsRed as calculated from Ref. 5.

b Photobleaching half-times during widefield illumination are listed as mean \pm s.e.m. for three independent replicates.

c For DsRed-Express2 and mKO2, all data except the $\mathrm{pK}_{\mathrm{a}}$ measurements were taken from Ref. 5.

$\mathrm{d}$ The photobleaching half-time for the green chromophore of E2-Red/Green cannot be compared directly to the photobleaching half-times of red chromophores because a different filter set was used.

\section{$A$ red and green dual-color derivative of DsRed-Express 2}

An FP that has both red and green fluorescence can be a useful "third color" for flow cytometry and fluorescence microscopy [13]. Fully mature wild-type DsRed is a mixture of molecules with either red- or green-emitting chromophores, but excitation of the green chromophores yields red fluorescence due to intra-tetramer FRET [14]. Our previous identification of rapidly maturing DsRed variants led to discovery of the $\mathrm{N} 42 \mathrm{H}$ mutation, which accelerates the maturation of wild-type DsRed more than 10-fold [6]. However, this mutation also increases the fraction of green-emitting chromophores, so that excitation with blue light now results in a substantial amount of green emission $[6,15]$.

We took advantage of the $\mathrm{N} 42 \mathrm{H}$ mutation to create a dual-color red and green derivative of DsRed-Express2. First, DsRed-Express2 was mutated to revert chromophore-facing mutations that had previously been introduced to minimize green fluorescence (Table 2). The $\mathrm{N} 42 \mathrm{H}$ mutation was then added to yield E2-Red/Green [GenBank: FJ498892]. When excited with blue light, E2Red/Green emits strongly at both red and green wavelengths (Figure 3A). When excited with yellow light to produce red fluorescence, E2-Red/Green is as bright as wild-type DsRed and more than twice as bright as DsRedExpress2 (Table 1). The red and green chromophores of E2-Red/Green mature quickly with half-times of $1.2 \mathrm{~h}$ and $0.4 \mathrm{~h}$, respectively (Figure 3C). Both chromophores are extremely photostable, even more so than the chromophores of DsRed-Express2 and EGFP (Figure 3D and 3E, Table 1).

To show that E2-Red/Green can be used as a third marker in conjunction with a green and a red FP, S. cerevisiae cells expressing DsRed-Express2, EGFP, E2-Red/Green, or no FP were grown separately and then pooled. This mixture was analyzed by flow cytometry with a 488-nm excitation laser. The three populations of fluorescent cells could be readily distinguished from one another and from the population of cells not expressing an FP (Figure 4).

\section{The new FPs are soluble and show low cytotoxicity and low phototoxicity in bacteria}

High-level expression in bacteria is the basis of convenient assays for FP solubility, cytotoxicity, and phototoxicity [5]. We used this approach to compare the new FPs to DsRed-Express 2 and to previously available orange FPs. One complication was that human codon-optimized $\mathrm{KO}$ is expressed poorly in bacteria, presumably due to unfavorable mRNA secondary structure near the start codon $[16,17]$. This problem was overcome by applying a method that had previously been used to optimize bacterial expression of DsRed-Express2 [5]. We screened bacteria expressing a library of modified $\mathrm{KO}$ genes that contained random combinations of the synonymous codons for amino acids 2-6. A sequence yielding colonies with bright fluorescence was designated $\mathrm{KO}^{*}$. Predicted secondary structures near the $5^{\prime}$ ends of the $\mathrm{KO}$ and $\mathrm{KO}^{*}$ mRNA sequences were compared using the program mfold [18]. The KO mRNA contained a predicted stable stem-loop involving the ribosomal binding site, and this stem-loop was disrupted in the $\mathrm{KO}^{*}$ mRNA (data not shown). This change may account for the stronger bacterial expression of $\mathrm{KO}^{*}$.

To confirm that the new FPs retain the high solubility of DsRed-Express2, we used a bacterial lysis and centrifugation assay in which aggregated oligomeric FPs are found in the pellet $[5,6]$. As with DsRed-Express2, only 3-5\% of 
the total fluorescence signal was found in the pellet with E2-Orange and E2-Red/Green (Figure 5A). By contrast, with $\mathrm{KO}^{*},>50 \%$ of the fluorescence signal was in the pellet (Figure 5A), indicating that this protein undergoes higher-order aggregation.

To test whether the new FPs retain the low bacterial cytotoxicity of DsRed-Express2, we examined colony size for E. coli cells expressing high levels of various FPs [5]. This experiment employed a regulatable promoter in which expression was derepressed using isopropyl- $\beta$-D-thiogalactopyranoside (IPTG). In the absence of IPTG, all of the colonies were large, as expected (Figure 5B). When IPTG was included in the medium, large colonies were seen for cells expressing DsRed-Express2, E2-Orange, or E2-Red/ Green, but much smaller colonies were seen for cells expressing mOrange 2 or $\mathrm{KO}^{*}$, indicating that the latter two proteins are cytotoxic (Figure $5 \mathrm{~B}$ ). We previously documented that $\mathrm{mKO} 2$ is also cytotoxic in bacteria [5]. SDSPAGE of whole-cell lysates confirmed that all of the FP genes except the original $\mathrm{KO}$ were expressed at comparable levels (Figure 5C). KO did not cause cytotoxicity in the

A
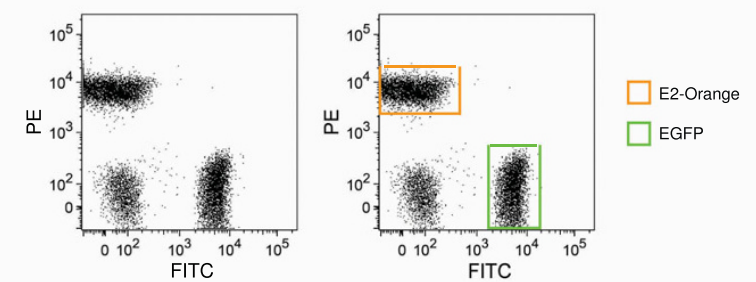
$\square$ EGFP
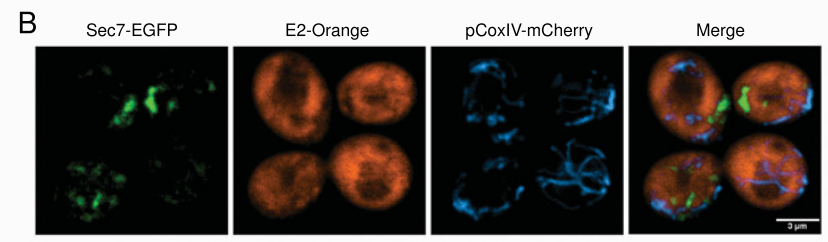

Figure 2

E2-Orange is useful as a second or third color. (A) Shown is a dot plot generated by flow cytometry of S. cerevisiae cells expressing E2-Orange, EGFP, or no FP. The three strains were grown separately, then pooled at equivalent cell concentrations and analyzed. Cells were excited using a 488$\mathrm{nm}$ laser, and orange and green fluorescence signals were detected using PE and FITC filter sets, respectively. On the right are the same data with E2-Orange-expressing cells boxed in orange and EGFP-expressing cells boxed in green. (B) A three-color yeast strain was generated with GFPlabeled Golgi, E2-Orange-labeled cytosol, and mCherrylabeled mitochondria. Cells were imaged using confocal microscopy. E2-Orange and GFP were immediately resolvable, and E2-Orange and mCherry were resolvable after linear unmixing, yielding clear three color images as shown in the overlay. presence of IPTG (Figure 5B) due to very low expression (Figure 5C). These data indicate that like DsRed-Express2, E2-Orange and E2-Red/Green exhibit unusually low cytotoxicity in bacteria.

Another experimentally relevant FP characteristic is phototoxicity during prolonged illumination. We used a bacterial cell survival assay [5] to measure the phototoxicity of E2-Orange, E2-Red/Green, and mKO2 during illumination through a Texas Red filter. This assay was not technically feasible with mOrange 2 or $\mathrm{KO}^{*}$ due to their slow maturation rates (Table 1). Control cells not expressing an FP showed nearly $100 \%$ survival (Figure 6A). E2-Orange and E2-Red/Green showed low to moderate phototoxicity with $91 \%$ and $69 \%$ survival, respectively. $\mathrm{mKO} 2$ showed high phototoxicity, with only $13 \%$ survival under the same conditions. These differences in phototoxicity were not due to different expression levels (Figure 6B). We conclude that E2-Orange and E2-Red/Green are likely to exhibit relatively mild phototoxicity during live-cell imaging.

\section{The new FPs show low cytotoxicity in HeLa cells}

To determine whether the DsRed-Express2 derivatives retain low cytotoxicity in mammalian cells, a fluorescence maintenance assay was performed [5]. HeLa cells were transiently transfected to express DsRed-Express2, E2Orange, E2-Red/Green, mOrange2, or KO under control of the strong CMV promoter, and the average brightness of fluorescent cells was monitored for five days using flow cytometry (Figure 7A). As previously observed, cells expressing DsRed-Express2 remained bright over the course of the experiment. Similarly, for cells expressing E2-Orange or E2-Red/Green, average cellular brightness was maximal at $48 \mathrm{~h}$ post-transfection and had decreased by $<10 \%$ at $120 \mathrm{~h}$ post-transfection. By contrast, for cells expressing mOrange 2 or $\mathrm{KO}$, average cellular brightness had decreased at $120 \mathrm{~h}$ by $40-50 \%$. The source of this decrease can be seen by comparing the flow cytometry data from $48 \mathrm{~h}$ and $120 \mathrm{~h}$. We assigned cells to groups, each of which represented a range of brightness values, and then plotted the percentage of cells in each group (Figure 7B). At $48 \mathrm{~h}$, the brightness of the transfected cells spanned from background fluorescence up to about 1000fold over background. At $120 \mathrm{~h}$, cells expressing high levels of DsRed-Express2, E2-Orange, or E2-Red/Green were detected with unchanged frequencies, but cells expressing high levels of mOrange 2 or $\mathrm{KO}$ were depleted from the population (Figure 7B). A similar loss of highly expressing cells was documented previously for mKO2 [5]. The combined data indicate that DsRed-Express 2 and its derivatives are noncytotoxic even at high expression levels, whereas previously available orange FPs show considerable cytotoxicity in mammalian cells. 

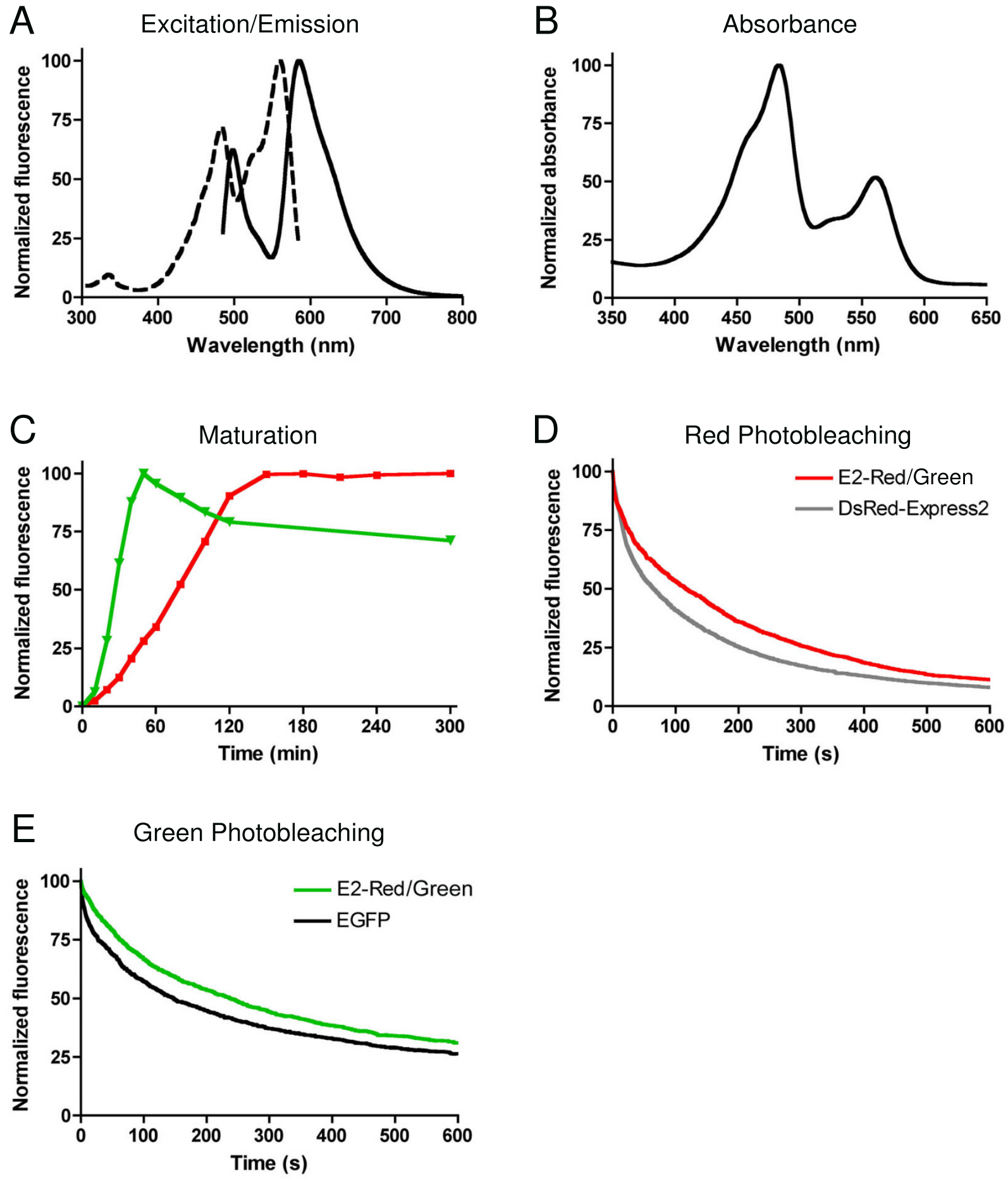

Figure 3

Fluorescence properties of E2-Red/Green. Shown are (A) excitation and emission and (B) absorbance spectra of E2-Red/ Green. (C) Maturation kinetics of green (green line) and red (red line) fluorescence of E2-Red/Green. For these measurements the green species was excited with $480 \pm 10 \mathrm{~nm}$ light and emission was recorded at $515 \pm 10 \mathrm{~nm}$, and the red species was excited with $540 \pm 10 \mathrm{~nm}$ light and emission was recorded at $590 \pm 10 \mathrm{~nm}$. Also shown are photobleaching kinetics for the red fluorescence of E2-Red/Green and DsRed-Express2 (D) and for the green fluorescence of E2-Red/Green and EGFP (E). The red and green photobleaching measurements were recorded using Texas Red and Endow GFP filter sets, respectively. 
Table 2: Substitutions made during the creation of E2-Red/ Green and E2-Orange.

\begin{tabular}{ll}
\hline Construct & Residues differing from DsRed-Express2 \\
\hline E2-Red/Green $^{\mathrm{a}}$ & Q42H, A44V, A2 I7T, A219G \\
E2-Orange $^{\mathrm{b}}$ & Q66T, V7IAc, S179Tc
\end{tabular}

a GenBank Accession Number F]498892

b GenBank Accession Number F]498891

c These mutations were identified during random mutagenesis. All other mutations resulted from targeted mutagenesis.

\section{Conclusion}

We predicted that it should be possible to modify the interior of DsRed-Express2 while preserving the optimized surface, thereby generating new noncytotoxic FPs with a variety of spectral properties [5]. The current study verifies that prediction. We incorporated mutations that had been reported to change the DsRed emission either to orange [7] or to a mixture of red and green $[6,15]$. The resulting DsRed-Express2 derivatives, E2-Orange and E2-Red/ Green, have favorable fluorescence properties including brightness, fast maturation, and high photostability. Importantly, these new FPs maintain the low cytotoxicity of DsRed-Express2 in bacterial and mammalian cells.

E2-Orange outperforms other available orange FPs by several criteria (Table 1). For comparison, mOrange 2 is photostable, but matures slowly and is very acid-sensitive [10] with a pKa of 7.5. mKO2 matures as quickly as E2-Orange, but is photolabile and phototoxic. Of the previously available FPs, KO is probably the best suited to whole-cell labe- ling because its slow maturation is offset by high brightness and reasonable photostability. Indeed, KO has been used to label murine hematopoietic stem cells [19] and to generate systemically labeled transgenic pigs [20]. How can we reconcile these results with the clear cytotoxicity of KO in our assays? We propose that reports of stable expression of KO and other cytotoxic FPs [21-23] reflect a threshold effect in which a cytotoxic FP is tolerated below a certain expression level. Thus, even if a cytotoxic FP is intrinsically bright, the observed signal may be relatively weak due to selection for low expression, and this low expression may cause residual cytotoxicity. By contrast, high-level expression of E2-Orange is likely to be maintained with little detrimental effect to the cells.

E2-Red/Green can be used as a "third color" in combination with existing green and red FPs in cases where three distinct cell populations each express a single type of FP. This type of application was suggested by Verkhusha and colleagues, who described a novel GFP variant, R10-3, in which a small fraction of the molecules generate a red chromophore [13]. E2-Red/Green should be easier to use as a dual-color cell label because its red emission is approximately 100-fold greater than that of R10-3. As an example of a potential application of E2-Red/Green, a standard 488-nm laser efficiently excites EGFP, DsRedExpress2, and E2-Red/Green, allowing three cell populations to be distinguished by flow cytometry. For fluorescence microscopy applications, images from green and red channels can be overlaid to distinguish cells expressing E2-Red/Green from those expressing either a pure
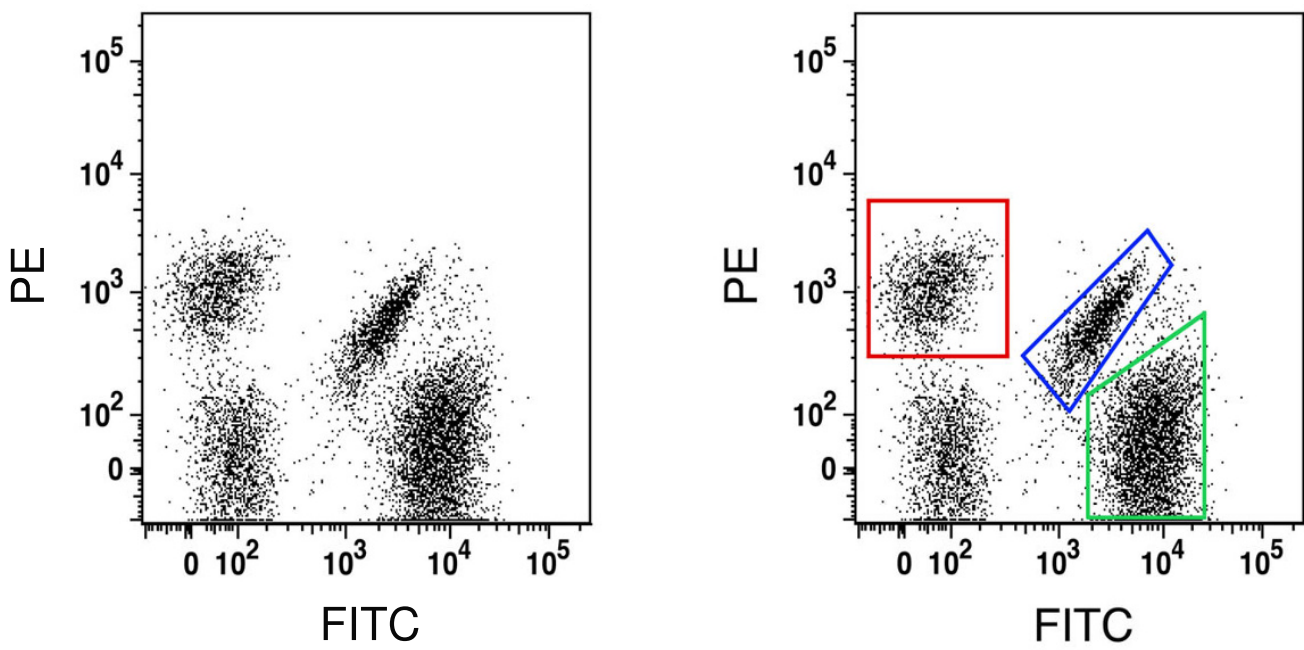

$\square$ DsRed-Express2

$\square$ E2-Red/Green EGFP

\section{Figure 4}

E2-Red/Green is a useful third color for flow cytometry. Shown is a dot plot generated by flow cytometry of S. cerevisiae cells expressing DsRed-Express2, E2-Red/Green, EGFP, or no FP. Cells were grown individually, pooled at equivalent cell concentrations, and analyzed by flow cytometry. Fluorescence was excited using a 488-nm laser, and red and green signals were detected using PE and FITC filter sets, respectively. On the right are the same data with DsRed-Express2-expressing cells boxed in red, E2-Red/Green-expressing cells boxed in blue, and EGFP-expressing cells boxed in green. 

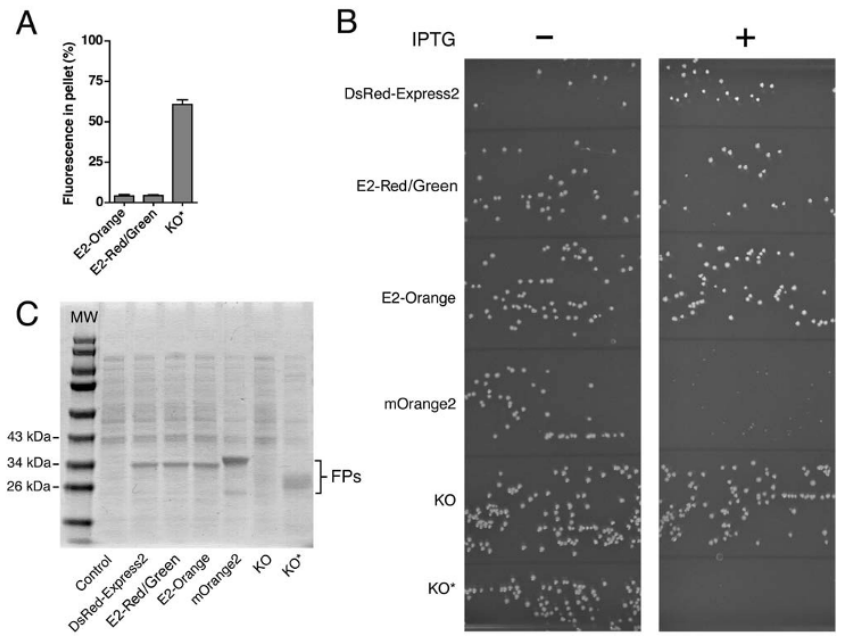

Figure 5

E2-Orange and E2-Red/Green do not form higherorder aggregates and are noncytotoxic to bacteria.

(A) To assay higher-order aggregation of oligomeric FPs, the percent fluorescence in the pellet fraction of a lysate from $E$. coli cells expressing E2-Orange, E2-Red/Green, or KO* was measured for eight independent replicates. Error bars represent s.e.m. (B) To measure bacterial cytotoxicity of FPs, $E$. coli DHIOB cells harboring the PREP4 repressor plasmid were transformed with $\mathrm{PQE}-60 \mathrm{NA}$ encoding DsRedExpress2, E2-Red/Green, E2-Orange, mOrange2, KO, or KO*. Equal volumes of transformation mixtures were plated onto adjacent sectors of plates under either repressing (no IPTG) or derepressing (I mM IPTG) conditions. Large colonies under derepressing conditions (right panel) indicate low cytotoxicity. (C) Quantitation of FP expression under derepressing conditions. Cells were grown to an $\mathrm{OD}_{600}$ of $\sim 0.6$ and then treated with I mM IPTG for $4 \mathrm{~h}$. Whole-cell lysates were separated using SDS-PAGE followed by staining with Coomassie Blue. Control cells were transformed with empty PQE-60NA.

green or a pure red FP. In this case, the cells expressing E2Red/Green will appear yellow in the overlaid images, while the other cells will appear either red or green.

Because E2-Orange and E2-Red/Green are tetramers, they are useful for whole-cell labeling rather than for making fusion proteins. Further modifications of the chromophore environment should enable us to generate additional whole-cell labels. For example, a far-red derivative of DsRed-Express 2 would be ideal for whole-animal imaging, and for multi-color labeling of cell populations in conjunction with E2-Orange.

\section{Methods}

\section{Characterization of FPs}

EGFP was obtained from Clontech. mOrange and mCherry were provided by R. Tsien (University of Califor-
A

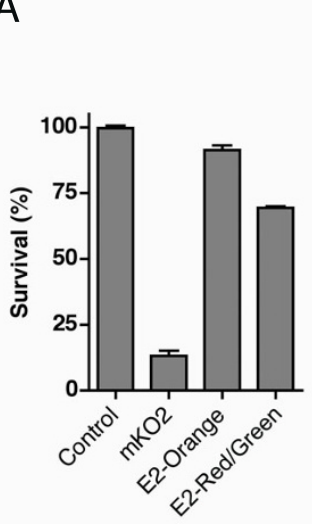

Figure 6

E2-Orange and E2-Red/Green show relatively mild phototoxicity. (A) E. coli cells were treated for $4 \mathrm{~h}$ with I mM IPTG to express either no FP (Control) or the indicated FP. Cells were then illuminated through a Texas Red (535$585 \mathrm{~nm}$ ) filter for $15 \mathrm{~min}$. In parallel, identical samples were not illuminated. Cells were then plated and grown overnight, and the percent survival was calculated based on colony number for the illuminated versus non-illuminated samples. Error bars represent s.e.m. (B) Quantitation of FP expression under the conditions of the phototoxicity experiment. Immediately before light treatment, aliquots of cells were taken for expression analysis. Whole-cell lysates were separated using SDS-PAGE followed by staining with Coomassie Blue.

nia at San Diego), and mOrange was modified by sitedirected mutagenesis to create mOrange $2 . \mathrm{mKO} 2$ was obtained as previously described [5]. Humanized KO was obtained by gene synthesis (GenScript). To create $\mathrm{KO}^{*}$, we replicated an earlier strategy that was used to enhance the bacterial expression of DsRed-Express2 [5]. Specifically, PCR of the humanized KO gene was performed using forward primers representing all possible synonymous codons for residues 2-6 (a mixture of 5'-TAGT AACCATGGTNTCNGTNATHAARCCCGAGATGAAGATGAAGTACTTC-3' and 5'-TAGTAACCATGGTNAGYGT NA THAARCCCGAGATGAAGA TGAAGTACTTC-3'). This PCR product was then digested with NcoI and NotI and subcloned into pQE-60NA [5], transformed into E. coli strain XL-1 Blue, and plated onto $\mathrm{LB}+100 \mu \mathrm{g} / \mathrm{ml}$ ampicilin lacking IPTG. After $12 \mathrm{~h}$ growth at $37^{\circ} \mathrm{C}$, cells were placed at room temperature for $10 \mathrm{~h}$ to allow for leaky expression. Colonies were then screened with the slide projector assay as previously described $[5,6]$ using a $520 \pm$ 20 excitation filter and a 550-nm longpass emission filter. Brightly fluorescent colonies were restreaked onto LB + $100 \mu \mathrm{g} / \mathrm{ml}$ ampicilin, and the brightest variant was sequenced and designated $\mathrm{KO}^{*}$. The sequence of codons $1-6$ in $\mathrm{KO}^{*}$ is 5'-ATGGTCAGTGTGATAAAA-3'. Secondary structure analysis of $\mathrm{KO}$ and $\mathrm{KO}^{*}$ was carried out using mfold [18]. The nucleotide sequences used for this analy- 

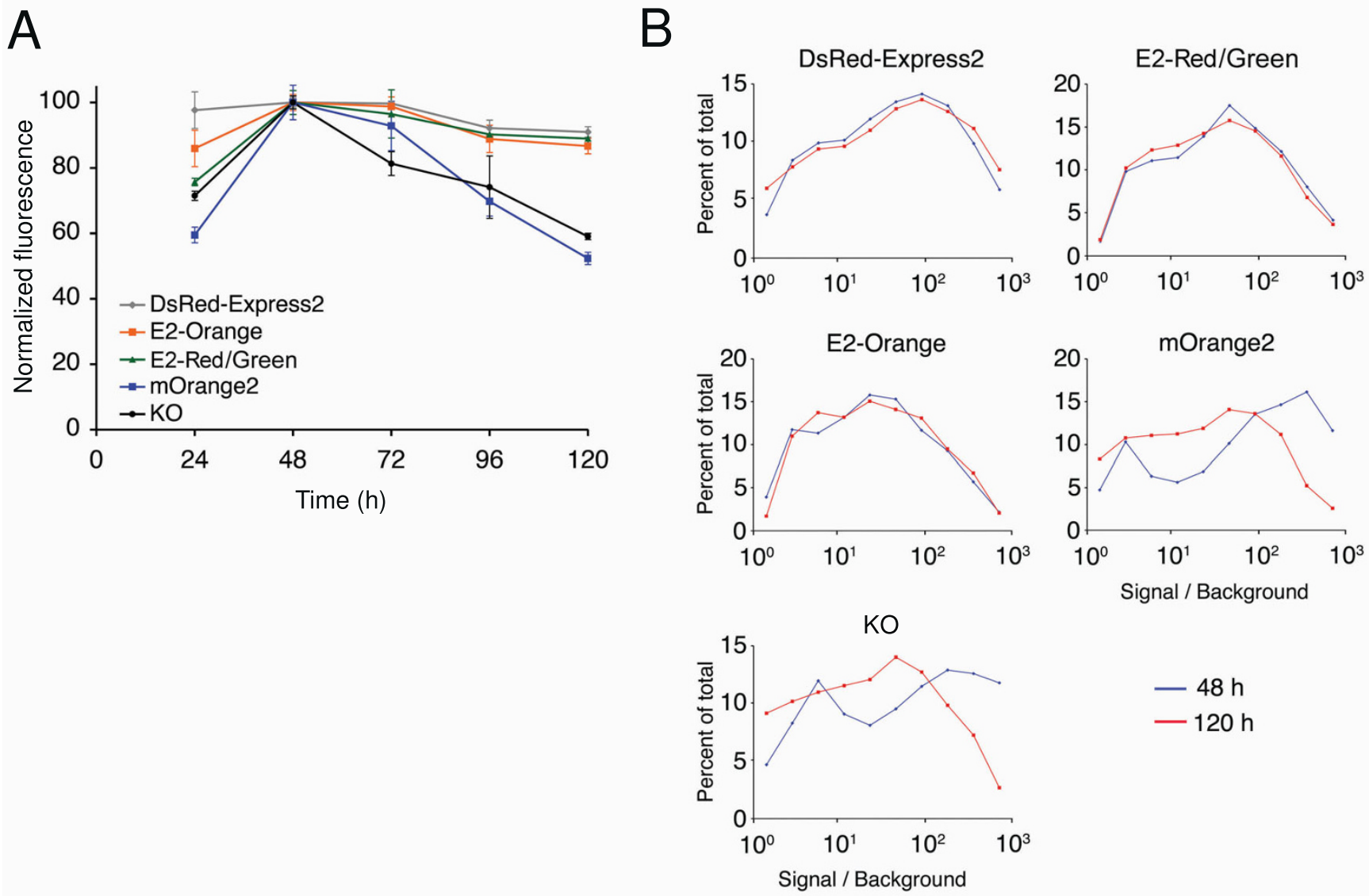

\section{Figure 7}

E2-Orange and E2-Red/Green are noncytotoxic to HeLa cells under conditions of standard high-level expression. (A) HeLa cells were transiently transfected in 24-well plates for constitutive high-level expression of the indicated FP. Three wells per FP per day were analyzed by flow cytometry, in parallel with untransfected cells, and the average brightness of the viable fluorescent cells was measured. The highest signal for a given FP was normalized to 100 units. Error bars represent s.e.m. (B) Fluorescence intensity distributions for the same data were analyzed for $48 \mathrm{~h}$ (blue) and $120 \mathrm{~h}$ (red) post-transfection. Each data point is a binned value that represents the percentage of cells with fluorescence in a range centered about the data point.

sis began 15 bp upstream of the ribosomal binding site and ended at codon 11 of the protein coding regions.

FPs were expressed, purified, and characterized for extinction coefficient and quantum yield as previously described $[5,6,24]$. The quantum yield of green chromophores was obtained using fluorescein in $0.1 \mathrm{M} \mathrm{NaOH}$ as a reference. The aggregation assay was carried out as previously described [5]. Photobleaching assays were carried out as previously described [5] using a Zeiss AxioPlan2 epifluorescence microscope with a 100-W mercury arc lamp, a 40× 0.75-NA air objective, and a Texas Red (535$585 \mathrm{~nm}$ excitation) or Endow GFP (450-490 nm excitation) filter set (Chroma).

$\mathrm{pK}_{\mathrm{a}}$ values were measured as follows. Purified FP was buffer exchanged and concentrated to $2 \mathrm{mg} / \mathrm{ml}$ into buffer containing $5 \mathrm{mM} \mathrm{Na}$ HEPES pH 8.0 and $100 \mathrm{mM} \mathrm{NaCl}$.
Aliquots were then adjusted to a final $\mathrm{pH}$ of 3.5-10.0 in $0.5 \mathrm{pH}$ unit increments by adding $5 \mu \mathrm{l}$ of a $1 \mathrm{M}$ adjustment buffer to $45 \mu \mathrm{l}$ of the FP sample. The adjustment buffers were $\mathrm{Na}^{+}$citrate ( $\left.\mathrm{pH} 3.5-5.5\right)$, Bis-Tris (pH 6-6.5), $\mathrm{Na}^{+}$HEPES ( $\mathrm{pH} 7.0-8.5$ ), and $\mathrm{Na}^{+}$CAPSO ( $\mathrm{pH}$ 9.0-10.0). Fluorescence measurements were carried out on a Tecan Safire $^{2}$ plate reader. Orange FPs were excited with $520 \pm 10$ $\mathrm{nm}$ light, and emission was recorded at $560 \pm 10 \mathrm{~nm}$. Red FPs were excited with $540 \pm 10 \mathrm{~nm}$ light, and emission was recorded at $590 \pm 10 \mathrm{~nm}$. Green FPs were excited with $480 \pm 10 \mathrm{~nm}$ light, and emission was recorded at $515 \pm 10$ $\mathrm{nm}$. Fluorescence values for a given FP were normalized to the highest observed emission, and the $\mathrm{pK}_{\mathrm{a}}$ was determined to be the point at which the fluorescence was half of the maximal signal.

Maturation experiments were carried out as previously described [24]. Briefly, E. coli DH10B cells harboring a 
given $\mathrm{FP}$ in the $\mathrm{PQE}-81$ vector were treated with $2 \mathrm{mM}$ IPTG for $15 \mathrm{~min}$ followed by inhibition of translation with $30 \mu \mathrm{g} / \mathrm{ml}$ kanamycin plus $17 \mu \mathrm{g} / \mathrm{ml}$ tetracyline. Fluorescence measurements were then recorded at regular time intervals using a Tecan Safire ${ }^{2}$ plate reader. For this assay, orange FPs were excited with $520 \pm 10 \mathrm{~nm}$ light, and emission was recorded at $560 \pm 10 \mathrm{~nm}$. Red FPs were excited with $540 \pm 10 \mathrm{~nm}$ light, and emission was recorded at $590 \pm 10 \mathrm{~nm}$. Green FPs were excited with 480 $\pm 10 \mathrm{~nm}$ light, and emission was recorded at $515 \pm 10 \mathrm{~nm}$.

\section{Library construction and screening}

Targeted mutations were introduced into the DsRedExpress2 gene by overlap extension PCR [25]. Combinatorial gene libraries with random mutations were built by error-prone PCR [26]. The PCR products were subcloned into pQE-60NA, transformed into E. coli strain DH10B, and analyzed for brightness and color using the slide projector assay.

\section{Bacterial cytotoxicity and phototoxicity assays}

For the E. coli colony size assay, DH10B cells harboring the pREP4 repressor plasmid were transformed with pQE60NA encoding the relevant FP. Equal volumes of cell mixture from the same transformation tube were plated either on an $\mathrm{LB}+50 \mu \mathrm{g} / \mathrm{ml}$ carbenicillin $+30 \mu \mathrm{g} / \mathrm{ml}$ kanamycin plate, or on a similar plate containing $1 \mathrm{mM}$ IPTG. Colonies were photographed after growth for $14 \mathrm{~h}$ at $37^{\circ} \mathrm{C}$. Phototoxicity was assayed as previously described [5].

\section{Fluorescence microscopy and flow cytometry of yeast}

To generate the three-color yeast strain, mCherry was subcloned into a plasmid carrying a yeast CoxIV presequence (pCoxIV) [6], and this plasmid was transformed into $S$. cerevisiae strain JK9-3da expressing Sec7-3xGFP [12]. This two-color strain was then transformed with a YIplac204 [27] derivative in which E2-Orange has been subcloned between the S. cerevisiae TPI1 promoter and the CYC1 terminator [28]. The resulting three-color cells were grown in quasi-synthetic dropout (QSD) media lacking leucine [12] and imaged using a Leica SP5 confocal microscope with a $63 \times$ oil objective. Linear unmixing was performed using Leica application suite (LAS-AF) software version 2.0, and image processing was performed using ImageJ http://rsb.info.nih.gov/ij/.

Yeast strains expressing a single FP were generated by transforming JK9-3da cells with a YIplac204 derivative containing the desired FP subcloned between the $S$. cerevisiae TPI1 promoter and the CYC1 terminator. Labeled yeast or untransformed control cells were grown individually in QSD media and pooled based on equivalent $\mathrm{OD}_{600}$ units. An LSRII flow cytometer (BD Biosciences) with a 488-nm laser was used with either FITC (515/50) or PE (585/15) emission filter sets to analyze cells. All data were processed using FloJo software (Treestar).

\section{HeLa cell cytotoxicity assay}

Transient transfection of HeLa cells and subsequent analysis by flow cytometry were carried out as previously described [5].

\section{Availability of E2-Orange and E2-Red/Green}

These plasmids can be obtained by request from Robert Keenan bkeenan@uchicago.edu.

\section{Authors' contributions}

RLS engineered E2-Orange and E2-Red/Green, and carried out all experiments. DB assisted with the mammalian tissue culture experiments. RLS, BSG, and RJK participated in conception of the project, design of the study, and manuscript preparation. All authors read and approved the final manuscript.

\section{Acknowledgements}

We thank D. Leclerc and R. Duggan at the University of Chicago Flow Cytometry Facility and V. Bindokas at the University of Chicago BSD Microscopy Core Facility for technical assistance. Thanks to S. Crosson for use of the spectropohotometer, to R. Tsien for providing mOrange and $m$ Cherry, and to $D$. Strongin for advice and feedback. We also thank members of the Glick and Keenan labs for feedback on the manuscript. This work was supported by NIH grants ROI EB008087 (to BSG and RJK) and T32 GM007I83 (to RLS).

\section{References}

I. Shaner NC, Patterson GH, Davidson MW: Advances in fluorescent protein technology. J Cell Sci 2007, I 20(Pt 24):4247-4260.

2. Tao W, Evans BG, Yao J, Cooper S, Cornetta K, Ballas CB, Hangoc $\mathrm{G}$, Broxmeyer HE: Enhanced green fluorescent protein is a nearly ideal long-term expression tracer for hematopoietic stem cells, whereas DsRed-Express fluorescent protein is not. Stem Cells 2007, 25(3):670-678.

3. Hadjantonakis AK, Macmaster S, Nagy A: Embryonic stem cells and mice expressing different GFP variants for multiple noninvasive reporter usage within a single animal. BMC Biotechnol 2002, 2: II.

4. Hirrlinger PG, Scheller A, Braun C, Quintela-Schneider M, Fuss B, Hirrlinger J, Kirchhoff F: Expression of reef coral fluorescent proteins in the central nervous system of transgenic mice. Mol Cell Neurosci 2005, 30(3):291-303.

5. Strack RL, Strongin DE, Bhattacharyya D, Tao W, Berman A Broxmeyer HE, Keenan RJ, Glick BS: A noncytotoxic DsRed variant for whole-cell labeling. Nat Methods 2008, 5(I I):955-957.

6. Bevis BJ, Glick BS: Rapidly maturing variants of the Discosoma red fluorescent protein (DsRed). Nat Biotechnol 2002, 20:83-87.

7. Shaner NC, Campbell RE, Steinbach PA, Giepmans BNG, Palmer AE, Tsien RY: Improved monomeric red, orange and yellow fluorescent proteins derived from Discosoma sp. red fluorescent protein. Nat Biotechnol 2005, 22:1567-1572.

8. Sakaue-Sawano A, Kurokawa H, Morimura T, Hanyu A, Hama H, Osawa H, Kashiwagi S, Fukami K, Miyata T, Miyoshi H, et al.: Visualizing spatiotemporal dynamics of multicellular cell-cycle progression. Cell 2008, I32(3):487-498.

9. Karasawa S, Araki T, Nagai T, Mizuno H, Miyawaki A: Cyan-emitting and orange-emitting fluorescent proteins as a donorl acceptor pair for fluorescence resonance energy transfer. Biochem I 2004, 381:307-312.

10. Shaner NC, Lin MZ, McKeown MR, Steinbach PA, Hazelwood KL, Davidson MW, Tsien RY: Improving the photostability of bright 
monomeric orange and red fluorescent proteins. Nat Methods 2008, 5(6):545-55I.

II. Shu X, Shaner NC, Yarbrough CA, Tsien RY, Remington S): Novel chromophores and buried charges control color in mFruits. Biochemistry 2006, 45:9639-9647.

12. Rossanese OW, Reinke CA, Bevis BJ, Hammond AT, Sears IB, O'Connor J, Glick BS: A role for actin, Cdclp and Myo2p in the inheritance of late Golgi elements in Saccharomyces cerevisiae . J Cell Biol 200I, I53:47-6I.

13. Mishin AS, Subach FV, Yampolsky IV, King W, Lukyanov KA, Verkhusha VV: The first mutant of the Aequorea victoria green fluorescent protein that forms a red chromophore. Biochemistry 2008, 47(16):4666-4673.

14. Baird GS, Zacharias DA, Tsien RY: Biochemistry, mutagenesis, and oligomerization of DsRed, a red fluorescent protein from coral. Proc Natl Acad Sci USA 2000, 97: I 1984-I 1989.

15. Wiehler J, von Hummel J, Steipe B: Mutants of Discosoma red fluorescent protein with a GFP-like chromophore. FEBS Lett 200I, 487:384-389.

16. Pfleger BF, Fawzi NJ, Keasling JD: Optimization of DsRed production in Escherichia coli: effect of ribosome binding site sequestration on translation efficiency. Biotechnol Bioeng 2005 , 92(5):553-558.

17. Sörensen M, Lippuner C, Kaiser T, Mißlitz A, Aebischer T, Bumann D: Rapidly maturing red fluorescent protein variants with strongly enhanced brightness in bacteria. FEBS Lett 2003 , 552: II0-II4.

18. Zuker M: Mfold web server for nucleic acid folding and hybridization prediction. Nucleic Acids Res 2003, 3 I ( I 3):3406-34I5.

19. Sanuki S, Hamanaka S, Kaneko S, Otsu M, Karasawa S, Miyawaki A, Nakauchi $\mathrm{H}$, Nagasawa T, Onodera M: A new red fluorescent protein that allows efficient marking of murine hematopoietic stem cells. J Gene Med 2008, I0(9):965-97I.

20. Matsunari $\mathrm{H}$, Onodera M, Tada N, Mochizuki $\mathrm{H}$, Karasawa S, Haruyama E, Nakayama N, Saito H, Ueno S, Kurome M, et al.: Transgenic-cloned pigs systemically expressing red fluorescent protein, Kusabira-Orange. Cloning Stem Cells 2008, I0(3):313-323.

21. Shcherbo D, Merzlyak EM, Chepurnykh TV, Fradkov AF, Ermakova GV, Solovieva EA, Lukyanov KA, Bogdanova EA, Zaraisky AG, Lukyanov $\mathrm{S}$, et al.: Bright far-red fluorescent protein for whole-body imaging. Nat Methods 2007, 4(9):74I-746.

22. Barolo S, Castro B, Posakony JW: New Drosophila transgenic reporters: insulated P-element vectors expressing fastmaturing RFP. Biotechniques 2004, 36(3):436-440. 442

23. Hoffman RM: The multiple uses of fluorescent proteins to visualize cancer in vivo. Nat Rev Cancer 2005, 5:796-806.

24. Strongin DE, Bevis B, Khuong N, Downing ME, Strack RL, Sundaram $K$, Glick BS, Keenan RJ: Structural rearrangements near the chromophore influence the maturation speed and brightness of DsRed variants. Protein Eng Des Sel 2007, 20(I I):525-534.

25. Ho SN, Hunt HD, Horton RM, Pullen JK, Pease LR: Site-directed mutagenesis by overlap extension using the polymerase chain reaction. Gene 1989, 77:5I-59.

26. Cadwell RC, Joyce GF: Mutagenic PCR. In PCR Primer A Laboratory Manual Edited by: Dieffenbach CW, Dveksler GS. Plainview: Cold Spring Harbor Laboratory Press; 1995:583-589.

27. Gietz RD, Sugino A: New yeast-Escherichia coli shuttle vectors constructed with in vitro mutagenized yeast genes lacking six-base pair restriction sites. Gene 1988, 74:527-534.

28. Reinke CA, Kozik P, Glick BS: Golgi inheritance in Saccharomyces cerevisiae depends on ER inheritance. Proc Natl Acad Sci USA 2004, 101:18018-18023.
Publish with Bio Med Central and every scientist can read your work free of charge

"BioMed Central will be the most significant development for disseminating the results of biomedical research in our lifetime. "

Sir Paul Nurse, Cancer Research UK

Your research papers will be:

- available free of charge to the entire biomedical community

- peer reviewed and published immediately upon acceptance

- cited in PubMed and archived on PubMed Central

- yours - you keep the copyright
BioMedcentral 